\title{
Design, synthesis, antimicrobial and cytotoxicity study on human colorectal carcinoma cell line of new 4,4'-(1,4-phenylene) bis(pyrimidin-2-amine) derivatives
}

Sanjiv Kumar ${ }^{1}$, Siong Meng Lim²,3, Kalavathy Ramasamy ${ }^{2,3}$, Vasudevan Mani ${ }^{4}$, Syed Adnan Ali Shah,6 and Balasubramanian Narasimhan ${ }^{{ }^{*}}$ (D)

\begin{abstract}
Background: Pyrimidine molecules attracted organic chemists very much due to their biological and chemotherapeutic importance. Their related fused heterocycles are of interest as potential bioactive molecules so, we have designed and prepared a new class of 4,4'-(1,4-phenylene)bis(pyrimidin-2-amine) molecules and screened for their in vitro antibacterial, antifungal and cytotoxicity studies.

Results: The structures of synthesized bis-pyrimidine molecules were confirmed by physicochemical and spectral means. The synthesized compounds were further evaluated for their in vitro biological potentials i.e. antimicrobial activity using tube dilution method and anticancer activity against human colorectal carcinoma (HCT116) cancer cell line by Sulforhodamine B assay.

Conclusions: The biological study demonstrated that compounds s7, s8, s11, s14, s16, s17 and $\mathbf{s 1 8}$ have shown more promising antimicrobial activity with best MIC values than the cefadroxil (antibacterial) and fluconazole (antifun$\mathrm{gal}$ ) and compound $\mathbf{s} \mathbf{3}$ found to have better anticancer activity against human colorectal carcinoma (HCT116) cancer cell line.
\end{abstract}

Keywords: Pyrimidine molecules, Design, Synthesis, Antimicrobial, Cytotoxicity, HCT116

\section{Background}

Among a wide variety of heterocyclic that have been explored for developing medicinally important molecules [1]. Pyrimidine derivatives attracted organic chemists very much due to their biological and chemotherapeutic importance especially the fused heterocycles are of interest as potential bioactive molecules. Pyrimidine derivatives are known to exhibit biological activities i.e. anticancer [2, 3], antiviral [4], anti-inflammatory [5], antimalarial [6], antibacterial [1, 7] and antifungal [8] etc. As pathogenic bacteria continuously evolve mechanisms

\footnotetext{
*Correspondence: naru2000us@yahoo.com

${ }^{1}$ Faculty of Pharmaceutical Sciences, Maharshi Dayanand University,

Rohtak 124001, India

Full list of author information is available at the end of the article
}

of resistance to currently used antibacterial, so the discovery of novel and potent antibacterial drugs is the best way to overcome bacterial resistance and develop effective therapies [9].

Cancer is one of the most serious health problems all over the world and one of the leading causes of death. Thus, in the past for several decades, researchers have been struggling to find effective clinical approaches for the treatment of cancer and search for novel anticancer agents. Recently, accumulating evidences have illustrated that heterocyclic derivatives are considered to be the most promising molecules as leads for the discovery of novel synthetic drugs. In particular, substituted pyrimidines, present in the cores of many physiologically active molecules, display interesting therapeutic properties, especially antitumor activities with different bio targets 
and mechanisms by means of inhibiting several enzymes as well as modulating the activity of many receptors [10]. Pyrimidine is found as a core structure in a large variety of compounds that exhibit important biological activity, specifically pyrimidines known to inhibit Pneumocystis carinii (pc), Toxoplasma gondii (tg) of tumour cell lines in culture and the activity is attributed to inhibition of dihydrofolate reductase (DHFR) [11]. 2,4-Disubstituted and 2,4,6-trisubstituted pyrimidines have shown potent anticancer activity as CDK inhibitors, TNF- $\alpha$ inhibitors, Abl tyrosine protein kinase inhibitors, PI-3 kinase inhibitors, Akt kinase inhibitors and cytokines inhibitors [12]. Design of pyrimidine molecules for antimicrobial and anticancer potentials based on literature is presented in Fig. 1. Selected marketed drug contains pyrimidine ring presented in Fig. 2 [13].

On the basis of these observations, we here in report the synthesis, in vitro antimicrobial and cytotoxicity activities of 4,4'-(1,4-phenylene)bis(pyrimidin-2-amine) derivatives.

\section{Results and discussion Chemistry}

Synthesis of the intermediate and target molecules was performed according to the reactions outlined in Scheme 1 (based on Claisen-Schmidt condensation). Initially, the bis-chalcone was prepared by the reaction of 1-(2,4-dichlorophenyl)ethanone and terephthalaldehyde. The cyclization of bis-chalcone (int-I) to yield bis-pyrimidine (int-II) was effected with guanidine hydrochloride. The reaction of bis-pyrimidine (int-II) with corresponding substituted aldehyde resulted in the formation of title compounds (s1-s18). The synthesized compounds were characterized by the determination of their physicochemical properties (Table 1) and spectral characteristics. The chemical structures of the synthesized 4,4'-(1,4-phenylene)bis(pyrimidin-2-amine) molecules (s1-s18) were established by ${ }^{1} \mathrm{H} /{ }^{13} \mathrm{C}-\mathrm{NMR}$, FT-IR, mass spectral studies and elemental analysis. The IR spectrum of bis-chalcone (I) showed the characteristic IR band at $1692.29 \mathrm{~cm}^{-1}$ which indicated the presence of $-\mathrm{C}=\mathrm{O}$ group and characteristic bands at 3089.33 and $1596.22 \mathrm{~cm}^{-1}$ indicated the presence of $\mathrm{C}-\mathrm{H}$ and $\mathrm{C}=\mathrm{C}$ group in aromatic ring, respectively. The existence of $\mathrm{Ar}-\mathrm{Cl}$ group in 3,3'-(1,4-phenylene) bis(1-(2,4-dichlorophenyl)prop-2-en-1-one) (I) was displayed by the existence $\mathrm{Ar}-\mathrm{Cl}$ stretches in the scale of $752.59 \mathrm{~cm}^{-1}$ and characteristic bands at 2829.17 and $1461.71 \mathrm{~cm}^{-1}$ indicated the presence of $\mathrm{C}-\mathrm{H}$ and $\mathrm{C}=\mathrm{C}$ group in alkyl chain, respectively. $6,6^{\prime}-(1,4-\mathrm{Phe}-$ nylene)bis(4-(2,4-dichlorophenyl)pyrimidin-2-amine) (II) showed the characteristic bands at 3089.99 and $1598.31 \mathrm{~cm}^{-1}$ for the presence of $\mathrm{C}-\mathrm{H}$ and $\mathrm{C}=\mathrm{C}$ group in aromatic ring, respectively and characteristic bands at 3363.97 and $1692.49 \mathrm{~cm}^{-1}$ indicated the presence of $-\mathrm{NH}_{2}$ and $\mathrm{N}=\mathrm{CH}$ str. The molecular structure of the intermediate-I and its cyclized products were further confirmed by proton-NMR spectral data. The ${ }^{1} \mathrm{H}-\mathrm{NMR}$ spectrum of intermediate-I showed two doublets at $7.59 \mathrm{ppm}(J=15.1 \mathrm{~Hz})$ and $8.06 \mathrm{ppm}$ $(J=15.1 \mathrm{~Hz})$ indicating that the $\mathrm{CH}=\mathrm{CH}$ group in the enone linkage is in a trans-conformation. The ${ }^{1} \mathrm{H}-\mathrm{NMR}$ spectrum of (II) showed a multiplet signals between 7.42 and $8.01 \delta \mathrm{ppm}$ confirming the cyclisation of the $3,3^{\prime}$-(1,4-phenylene) bis(1-(2,4-dichlorophenyl)prop2-en-1-one) (I) to give 6,6'-(1,4-phenylene)bis(4-(2,4dichlorophenyl)pyrimidin-2-amine) (II). The ${ }^{1} \mathrm{H}$-NMR spectrum of intermediate-II showed a sharp singlet at $7.09 \delta \mathrm{ppm}$ due to the $\mathrm{NH}_{2}$ protons and it also showed a sharp singlet at $7.85 \delta \mathrm{ppm}$ due to $\mathrm{HC}=\mathrm{C}$ group, which confirmed the cyclization of the bis-chalcone into a bis-pyrimidine ring. The IR stretching vibrations at $733.88-750.53 \mathrm{~cm}^{-1}$ in the spectral data of synthesized derivatives (s1-s18) displayed the presence of halogen group $(\mathrm{Ar}-\mathrm{Cl})$ on the aromatic nucleus substituted at the ortho, meta and para-position. The existence of $\mathrm{Ar}-\mathrm{NO}_{2}$ functional group in compounds s3, s6 and s7 was displayed by the existence of symmetric $\mathrm{Ar}-\mathrm{NO}_{2}$ stretches in the scale of $1372.55-1373.82 \mathrm{~cm}^{-1}$. The existence of an arylalkyl ether group $\left(\mathrm{Ar}-\mathrm{OCH}_{3}\right)$ in compounds, s5, s9, s10, s12 and s13 are established by the existence of an IR absorption band around $3088.33-3089.60 \mathrm{~cm}^{-1}$. The impression of IR stretching vibration at $3088.93-2972.97 \mathrm{~cm}^{-1}$ and $1599.67-$ $1595.05 \mathrm{~cm}^{-1}$ in the spectral data of synthesized derivatives (s1-s18) specified the existence of $\mathrm{C}-\mathrm{H}$ and $\mathrm{C}=\mathrm{C}$ group, respectively. The appearance of IR stretching $1698.99-1663.17 \mathrm{~cm}^{-1}$ in the spectral data of synthesized derivatives (s1-s18) specified the existence of $\mathrm{N}=\mathrm{CH}$ group. The impression of IR absorption band at $3461.41-3345.04 \mathrm{~cm}^{-1}$ in the spectral data s2, s4, s13 and s15 displayed the presence of $\mathrm{Ar}-\mathrm{OH}$ group on the aromatic ring at ortho and para position. The multiplet signals between 6.77 and $8.34 \delta \mathrm{ppm}$ in proton-NMR spectra is indicative of aromatic proton of synthesized derivatives. The compounds, s5, s9, s10, s12 and s13 showed singlet at $3.84-3.85 \delta \mathrm{ppm}$ due to the existence of $\mathrm{OCH}_{3}$ of $\mathrm{Ar}-\mathrm{OCH}_{3}$. The synthesized compounds showed singlet at 9.01-10.05 $\delta \mathrm{ppm}$ due to the existence of $\mathrm{N}=\mathrm{CH}$ in pyrimidine ring. Compounds showed singlet at $10.00-10.15 \delta \mathrm{ppm}$ due to the existence of $-\mathrm{CH}$ in pyrimidine ring. Compound $\mathbf{s} 8$ showed singlet at 3.04 $\delta \mathrm{ppm}$ due to existence of $-\mathrm{N}\left(\mathrm{CH}_{3}\right)_{2}$ at the para position. The compound s16 showed quadrate at 3.38-3.49 $\delta \mathrm{ppm}$ and triplet at $1.07-1.15 \delta \mathrm{ppm}$ due to presence of $-\mathrm{N}\left(\mathrm{C}_{2} \mathrm{H}_{5}\right)_{2}$ at para position. The 


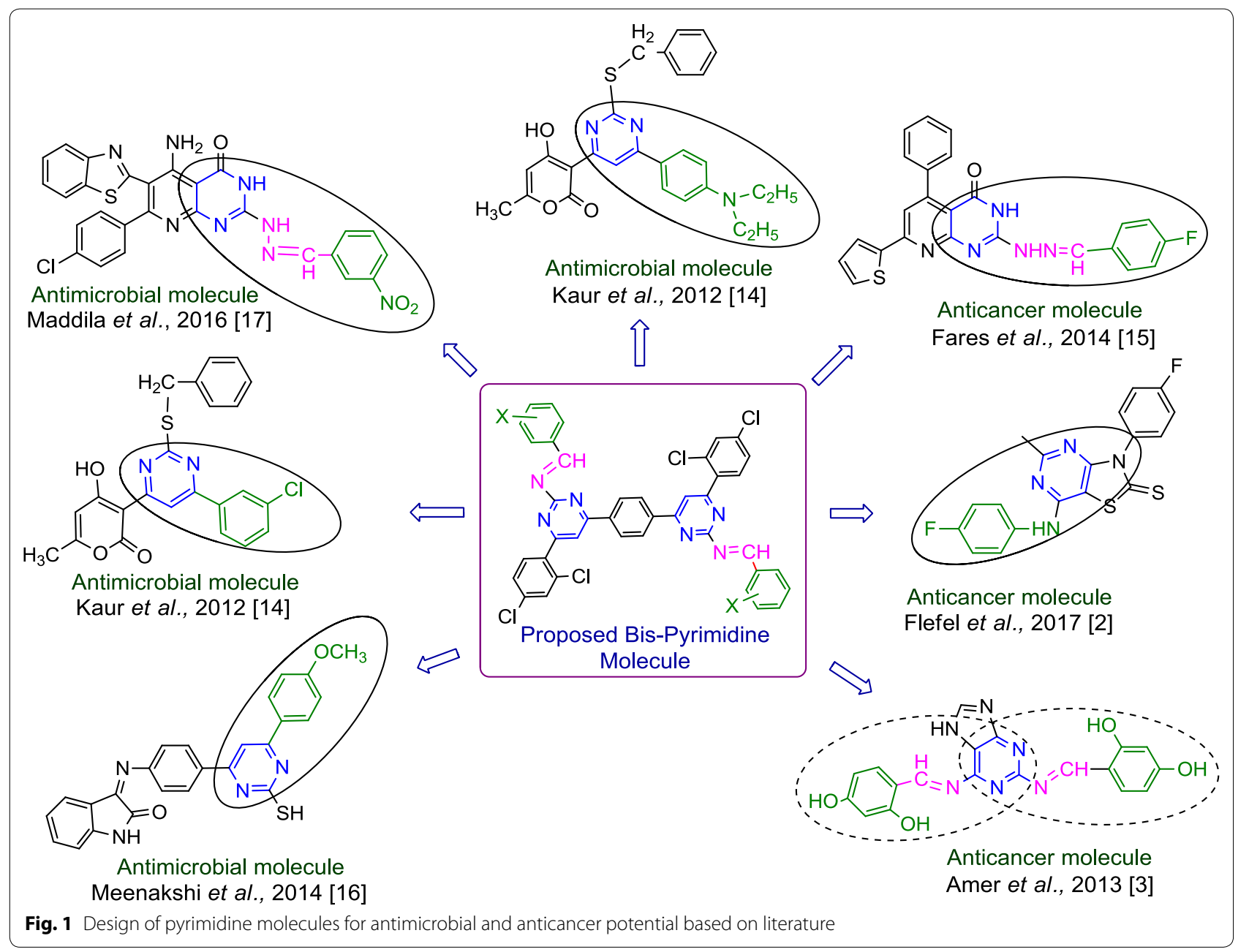

${ }^{13} \mathrm{C}-\mathrm{NMR}$ spectral data and elemental analysis studies of the synthesized pyrimidine derivatives were found within $\pm 0.4 \%$ of the theoretical results of synthesized compounds are given in the "Experimental section".

\section{In vitro antimicrobial activity}

Antimicrobial screening of synthesized 4,4'-(1,4-phenylene)bis(pyrimidin-2-amine) molecules against Gram positive and Gram negative bacterial and fungal strains was done by tube dilution technique. Antimicrobial activity results indicated (Table 2) particularly; compounds, s7, s8, s11, s14, s16, s17 and s18 have shown more promising antimicrobial activity as compared to standard drugs cefadroxil (antibacterial) and fluconazole (antifungal) while other derivatives are moderately active. In the case of Gram positive bacterial study, compound s11 $\left(\mathrm{MIC}_{s a}=0.14 \mu \mathrm{mol} / \mathrm{mL}, \mathrm{MIC}_{b c}=0.07 \mu \mathrm{mol} / \mathrm{mL}\right)$ was found to be most potent one against $S$. aureus and $B$. cereus. In the case of Gram negative bacterial study, compound s7 and s18 displayed appreciable antibacterial activity against Providencia rettgeri with MIC value of $0.08 \mu \mathrm{mol} / \mathrm{mL}$. Compound $\mathbf{s 8}\left(\mathrm{MIC}_{p a}=0.15 \mu \mathrm{mol} /\right.$ $\mathrm{mL}$ ) exhibited excellent activity against Pseudomonas aeruginosa and compound s11 showed good antibacterial activity against Salmonella typhi and Escherichia coli with the MIC values of 0.29 and $0.23 \mu \mathrm{mol} / \mathrm{mL}$, respectively. The antifungal screening results demonstrated that compounds s11 displayed appreciable antifungal activity against Aspergillus niger and Aspergillus flavus with the MIC values of 0.58 and $0.14 \mu \mathrm{mol} / \mathrm{mL}$, respectively. Compounds, s14 and s17 $\left(\mathrm{MIC}_{a f}=0.31 \mu \mathrm{mol} / \mathrm{mL}\right)$ were found to be most potent ones against Aspergillus fumigatus and compound $\mathbf{s 1 6}\left(\mathrm{MIC}_{a f}=0.14 \mu \mathrm{mol} / \mathrm{mL}\right)$ was found to be most effective one against Aspergillus flavus. The antimicrobial screening results of synthesized molecules (s7, s8, s11, s14, s16, s17 and s18) have more than standard drugs and may be used as a lead compound to discover novel antimicrobial scaffolds. 
<smiles>COc1cc(NS(=O)(=O)c2ccc(N)cc2)nc(OC)n1</smiles>

Sulfadimethoxine<smiles>Nc1nc(=O)[nH]cc1F</smiles>

Flucytosine<smiles>Cc1ccc(Cc2cnc(NCCCCc3ncc(Br)cc3C)[nH]c2=O)cn1</smiles>

Temelastine<smiles>O=c1[nH]c(=O)n(C2CCCO2)cc1F</smiles>

Tegafur<smiles>Nc1nc(N)c2nc(-c3ccccc3)c(N)nc2n1</smiles>

Triamterene<smiles>COc1cc(Cc2cnc(N)nc2N)cc(OC)c1Br</smiles>

Brodimoprim<smiles>Cc1cn(C2CCC(CO)O2)c(=O)nc1N</smiles>

Zalcitabine<smiles>Cc1ccccc1N1C(=O)c2cc(N)ccc2NC1CF</smiles>

Afloqualone<smiles>O=C(O)c1cc(=O)[nH]c(=O)[nH]1</smiles>

Orotic Acid<smiles>Cc1nc2n(c(=O)c1CCN1CCC(c3noc4c(F)cccc34)CC1)CCCC2</smiles>

Risoperidone

Fig. 2 Selected marketed drug contains pyrimidine ring

\section{In vitro anticancer activity}

The in vitro anticancer activity of synthesized 4,4'-(1,4-phenylene)bis(pyrimidin-2-amine) molecules was carried out against human colorectal cancer cell line [HCT-116 (ATCC CCL-247)] and compared with 5 -fluorouracil (reference drug) and the results of anticancer studies have been presented in Table 3, Fig. 3. Anticancer screening results revealed that in general pyrimidine exhibited good anticancer potential against human colorectal cancer cell line, especially, compound s3 $\left(\mathrm{IC}_{50}=1.16 \mu \mathrm{mol} / \mathrm{mL}\right)$ displayed anticancer activity comparable to the reference drug 5-fluorouracil $\left(\mathrm{IC}_{50}=0.83 \mu \mathrm{mol} / \mathrm{mL}\right)$.

\section{Structure-activity relationship}

From the in vitro antibacterial, antifungal and anticancer results, the structure-activity relationship of synthesized 4,4'-(1,4-phenylene)bis(pyrimidin-2-amine) molecules (SAR, are presented in Fig. 4).
From structure activity relationship study, we may conclude that different structural requirements are required for a compound to be effective against different targets. The aforementioned facts are supported by the earlier research findings $[3,14,16,17]$.

\section{Experimental section}

Preliminary materials (glasswares, chemicals etc.) for the research work were obtained from commercial sources [Loba Chemie, Pvt Ltd. Mumbai, India; Central Drug House (CDH) Pvt. Ltd., New Delhi, India and HiMedia Laboratory Pvt. Ltd., Delhi, India] used without further purification. All reactions were monitored by thin-layer chromatography on $0.25 \mathrm{~mm}$ silica gel (Merck) plates, using benzene, chloroform: methanol as mobile phase and spots were observed by exposure to iodine vapours. Melting points of synthesized 4,4'-(1,4-phenylene) bis(pyrimidin-2-amine) molecules was determined in open capillary tube technique. Mass spectra of the 


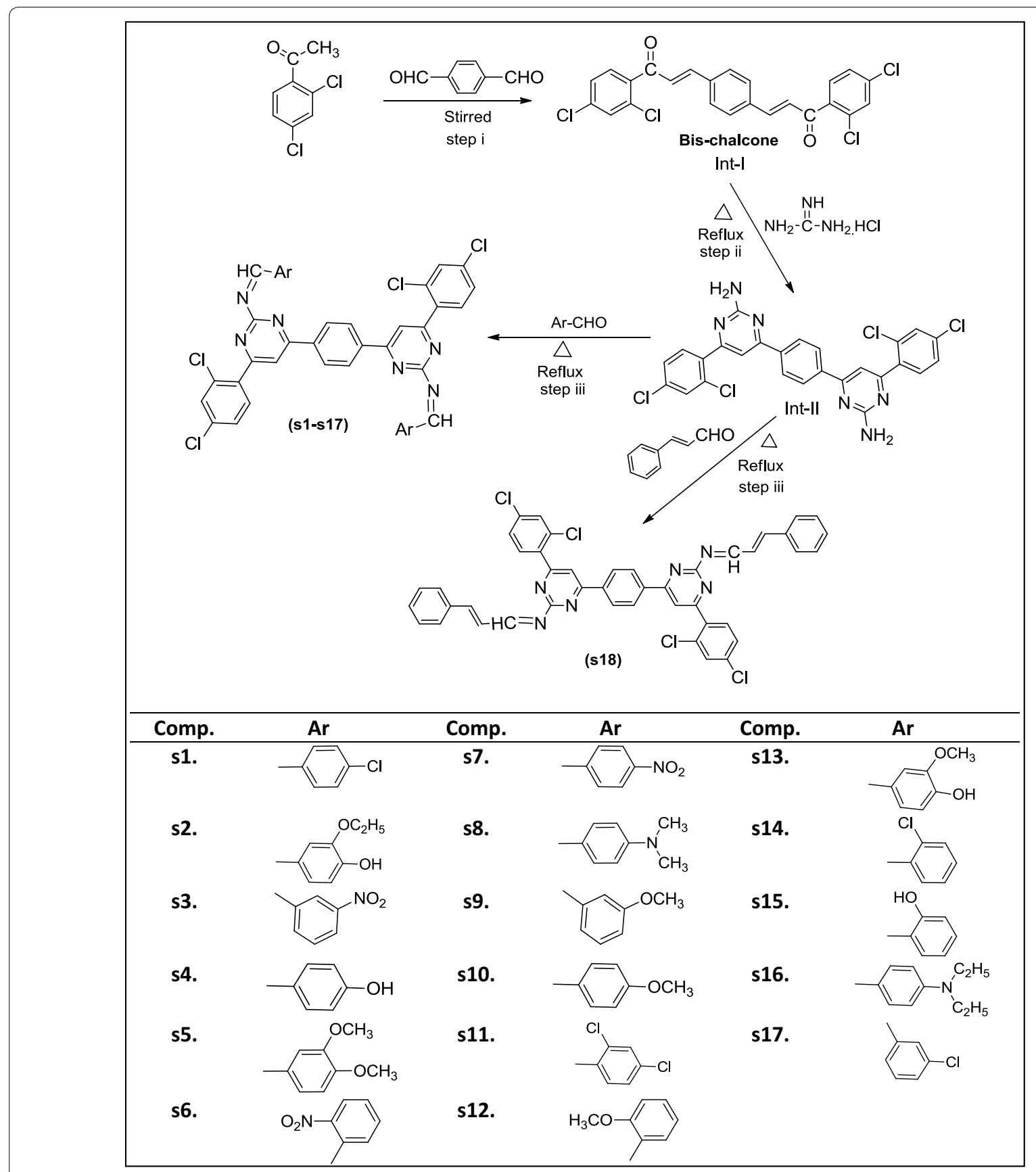

Scheme 1 Synthesis of bis-pyrimidine molecules of 4,4'-(1,4-phenylene)bis(pyrimidin-2-amine)

synthesized compounds were recorded on Waters Micromass Q-ToF Micro instrument. An infrared spectrum (IR) was recorded (KBr-pellets) in Bruker 12060280, Software: OPUS 7.2.139.1294 spectrometer. ${ }^{1} \mathrm{H}-\mathrm{NMR}$ and ${ }^{13} \mathrm{C}$-NMR were recorded at 600 and $150 \mathrm{MHz}$ respectively on Bruker Avance III 600 NMR spectrometer by appropriate deuterated solvents. The results are conveyed in parts per million $(\delta, \mathrm{ppm})$ downfield from tetramethyl silane (internal standard). ${ }^{1} \mathrm{H}-\mathrm{NMR}$ spectral details of the synthesized derivatives are represented with multiplicity like singlet (s); doublet (d); triplet $(\mathrm{t})$; quartet (q); multiplet $(\mathrm{m})$ and the number hydrogen ion. Elemental analysis of the synthesized 4,4'-(1,4-phenylene) bis(pyrimidin-2-amine) molecules was obtained by Perkin-Elmer $2400 \mathrm{C}, \mathrm{H}$ and $\mathrm{N}$ analyzer. All the compounds 
Table 1 Physicochemical properties of the synthesized bis-pyrimidine molecules

\begin{tabular}{|c|c|c|c|c|c|c|}
\hline $\begin{array}{c}\text { Comp. } \\
\text { no }\end{array}$ & Structure & $\begin{array}{l}\text { Molecular } \\
\text { formula }\end{array}$ & Color & M.pt. $\left({ }^{\circ} \mathrm{C}\right)$ & $\mathrm{R}_{f}$ value & $\%$ Yield \\
\hline s1. & & $\mathrm{C}_{40} \mathrm{H}_{22} \mathrm{Cl}_{6} \mathrm{~N}_{6}$ & $\begin{array}{l}\text { Dark } \\
\text { yellow }\end{array}$ & $133-135$ & 0.46 & 85.45 \\
\hline s2. & & $\mathrm{C}_{44} \mathrm{H}_{32} \mathrm{Cl}_{4} \mathrm{~N}_{6} \mathrm{O}_{4}$ & $\begin{array}{l}\text { Light } \\
\text { yellow }\end{array}$ & $113-115$ & 0.25 & 75.56 \\
\hline s3. & & $\mathrm{C}_{40} \mathrm{H}_{22} \mathrm{Cl}_{4} \mathrm{~N}_{8} \mathrm{O}_{4}$ & $\begin{array}{l}\text { Cream } \\
\text { yellow }\end{array}$ & $140-142$ & 0.31 & 69.03 \\
\hline s4. & & $\mathrm{C}_{40} \mathrm{H}_{24} \mathrm{Cl}_{4} \mathrm{~N}_{6} \mathrm{O}_{2}$ & $\begin{array}{l}\text { Pure } \\
\text { yellow }\end{array}$ & $133-135$ & 0.26 & 82.56 \\
\hline s5. & & $\mathrm{C}_{44} \mathrm{H}_{32} \mathrm{Cl}_{4} \mathrm{~N}_{6} \mathrm{O}_{4}$ & $\begin{array}{l}\text { Dark } \\
\text { yellow }\end{array}$ & $145-147$ & 0.20 & 69.23 \\
\hline s6. & & $\mathrm{C}_{40} \mathrm{H}_{22} \mathrm{Cl}_{4} \mathrm{~N}_{8} \mathrm{O}_{4}$ & $\begin{array}{l}\text { Medallion } \\
\text { yellow }\end{array}$ & $146-148$ & 0.35 & 70.00 \\
\hline s7. & & $\mathrm{C}_{40} \mathrm{H}_{22} \mathrm{Cl}_{4} \mathrm{~N}_{8} \mathrm{O}_{4}$ & $\begin{array}{l}\text { Light } \\
\text { yellow }\end{array}$ & $142-144$ & 0.32 & 75.65 \\
\hline s8. & & $\mathrm{C}_{44} \mathrm{H}_{34} \mathrm{Cl}_{4} \mathrm{~N}_{8}$ & $\begin{array}{l}\text { Light } \\
\text { yellow }\end{array}$ & $123-125$ & 0.39 & 78.12 \\
\hline s9. & & $\mathrm{C}_{42} \mathrm{H}_{28} \mathrm{Cl}_{4} \mathrm{~N}_{6} \mathrm{O}_{2}$ & $\begin{array}{l}\text { Honey } \\
\text { yellow }\end{array}$ & $129-131$ & 0.25 & 65.01 \\
\hline
\end{tabular}


Table 1 (continued)

\begin{tabular}{|c|c|c|c|c|c|c|}
\hline $\begin{array}{c}\text { Comp. } \\
\text { no }\end{array}$ & Structure & $\begin{array}{c}\text { Molecular } \\
\text { formula }\end{array}$ & Colour & M.pt. $\left({ }^{\circ} \mathrm{C}\right)$ & $\mathrm{R}_{f}$ value & $\%$ Yield \\
\hline s10. & & $\mathrm{C}_{42} \mathrm{H}_{28} \mathrm{Cl}_{4} \mathrm{~N}_{6} \mathrm{O}_{2}$ & $\begin{array}{l}\text { Pure } \\
\text { yellow }\end{array}$ & $124-126$ & 0.23 & 80.45 \\
\hline s11. & & $\mathrm{C}_{40} \mathrm{H}_{20} \mathrm{Cl}_{8} \mathrm{~N}_{6}$ & $\begin{array}{l}\text { Lemon } \\
\text { yellow }\end{array}$ & $80-82$ & 0.21 & 79.34 \\
\hline $\mathrm{s} 12$. & & $\mathrm{C}_{42} \mathrm{H}_{28} \mathrm{Cl}_{4} \mathrm{~N}_{6} \mathrm{O}_{2}$ & $\begin{array}{l}\text { Light } \\
\text { yellow }\end{array}$ & $134-135$ & 0.58 & 82.23 \\
\hline s13. & & $\mathrm{C}_{42} \mathrm{H}_{28} \mathrm{Cl}_{4} \mathrm{~N}_{6} \mathrm{O}_{4}$ & $\begin{array}{l}\text { Pure } \\
\text { yellow }\end{array}$ & $129-131$ & 0.41 & 89.45 \\
\hline s14. & & $\mathrm{C}_{40} \mathrm{H}_{22} \mathrm{Cl}_{6} \mathrm{~N}_{6}$ & $\begin{array}{l}\text { Medallion } \\
\text { yellow }\end{array}$ & $56-58$ & 0.43 & 85.56 \\
\hline s15. & & $\mathrm{C}_{40} \mathrm{H}_{24} \mathrm{Cl}_{4} \mathrm{~N}_{6} \mathrm{O}_{2}$ & $\begin{array}{l}\text { Dark } \\
\text { yellow }\end{array}$ & $79-81$ & 0.50 & 87.23 \\
\hline s16. & & $\mathrm{C}_{48} \mathrm{H}_{42} \mathrm{Cl}_{4} \mathrm{~N}_{8}$ & $\begin{array}{l}\text { Cream } \\
\text { yellow }\end{array}$ & $75-77$ & 0.37 & 66.33 \\
\hline s17. & & $\mathrm{C}_{40} \mathrm{H}_{22} \mathrm{Cl}_{6} \mathrm{~N}_{6}$ & $\begin{array}{l}\text { Dark } \\
\text { yellow }\end{array}$ & $56-58$ & 0.57 & 68.12 \\
\hline s18. & & $\mathrm{C}_{44} \mathrm{H}_{28} \mathrm{Cl}_{4} \mathrm{~N}_{6}$ & $\begin{array}{l}\text { Light } \\
\text { yellow }\end{array}$ & $63-65$ & 0.50 & 62.23 \\
\hline
\end{tabular}


Table 2 Antimicrobial activity results of synthesized bis-pyrimidine molecules

\begin{tabular}{|c|c|c|c|c|c|c|c|c|c|}
\hline \multirow[t]{4}{*}{ Compound no. } & \multicolumn{6}{|c|}{ Antimicrobial activity $(\mathrm{MIC}=\mu \mathrm{mol} / \mathrm{mL}$ ) } & \multicolumn{3}{|c|}{ Fungal species } \\
\hline & \multicolumn{6}{|c|}{ Bacterial species } & \multirow[b]{3}{*}{ A.N. } & \multirow[b]{3}{*}{ A. $F^{\mathrm{a}}$} & \multirow[b]{3}{*}{ A.F. ${ }^{\mathrm{b}}$} \\
\hline & \multicolumn{2}{|c|}{ Gram positive } & \multicolumn{4}{|c|}{ Gram negative } & & & \\
\hline & S.A. & B.C. & S.T. & P.A. & E.C. & P.R. & & & \\
\hline s1. & 0.16 & 0.08 & - & - & 0.25 & 0.16 & 0.63 & 1.26 & - \\
\hline s2. & 2.36 & 1.18 & - & 2.36 & - & 2.36 & 2.36 & 1.18 & 0.59 \\
\hline s3. & 0.61 & 0.61 & - & - & - & 0.15 & 0.61 & 1.22 & 0.61 \\
\hline s4. & 0.16 & 0.08 & 0.33 & 0.16 & 0.26 & 0.16 & 2.63 & 0.66 & 0.16 \\
\hline s5. & 0.59 & 0.59 & - & - & - & 0.15 & - & 1.18 & 1.18 \\
\hline s6. & - & 0.15 & 2.44 & 1.22 & 0.61 & - & 2.44 & 0.61 & 0.15 \\
\hline s7. & 0.61 & - & 1.22 & 0.61 & 2.44 & 0.08 & - & 1.22 & - \\
\hline s8. & - & 1.23 & 0.31 & 0.15 & 0.61 & - & 2.46 & 0.61 & 0.15 \\
\hline s9. & 0.16 & 0.08 & 0.32 & 0.16 & 0.25 & 0.16 & - & - & 0.32 \\
\hline s10. & 1.27 & 0.63 & 1.27 & 2.54 & - & 0.63 & 0.63 & 1.27 & 0.32 \\
\hline s11. & 0.14 & 0.07 & 0.29 & 1.16 & 0.23 & 0.14 & 0.58 & - & 0.14 \\
\hline s12. & 1.27 & 0.63 & - & 2.54 & - & 0.63 & - & 1.27 & 0.32 \\
\hline s13. & 1.22 & 0.61 & - & - & 1.22 & 0.61 & 1.22 & 1.22 & - \\
\hline s14. & 2.51 & 1.26 & - & 1.26 & - & 2.51 & - & 0.31 & 0.63 \\
\hline s15. & 0.66 & 0.66 & - & - & 0.66 & 0.16 & 0.66 & 0.33 & - \\
\hline s16. & 0.57 & 0.57 & - & - & 0.57 & 0.14 & 2.30 & 0.57 & 0.14 \\
\hline s17. & - & 0.16 & 2.51 & 1.26 & 0.63 & 1.26 & 0.63 & 0.31 & 1.26 \\
\hline s18. & 0.64 & - & 1.28 & 0.64 & 2.56 & 0.08 & 1.28 & - & 0.32 \\
\hline Acetone & NA & NA & NA & NA & NA & NA & NA & NA & NA \\
\hline Broth control & $N G$ & $N G$ & $N G$ & $N G$ & $N G$ & NG & $N G$ & $N G$ & $N G$ \\
\hline Std. & $0.34^{c}$ & $0.34^{c}$ & $0.68^{c}$ & $0.68^{c}$ & $0.34^{c}$ & $0.68^{c}$ & $0.82^{d}$ & $0.82^{d}$ & $0.82^{\mathrm{d}}$ \\
\hline
\end{tabular}

S.A.: Staphylococcus aureus; B.C.: Bacillus cereus; S.T.: Salmonella typhi; P.A.: Pseudomonas aeruginosa; E.C.: Escherichia coli; P.R.: Providencia rettgeri; A.N.: Aspergillus niger

a A.F.: Aspergillus fumigatus; ${ }^{\mathrm{b}}$ A.F.: Aspergillus flavus; Resistant (-); NA no activity, NG No growth

Std.: Cefadroxil'; Fluconazole ${ }^{\text {d }}$

gave $\mathrm{C}, \mathrm{H}$ and $\mathrm{N}$ analysis within $\pm 0.4 \%$ of the theoretical results.

\section{General procedure for the synthesis of 4,4'-(1,4-phenylene)bis(pyrimidin-2-amine) derivatives (s1-s18)}

\section{Step i: Synthesis of 3,3'-(1,4-phenylene)}

bis(1-(2,4-dichlorophenyl)prop-2-en-1-one) (Int-I)

A mixture of 1-(2,4-dichlorophenyl)ethanone $(0.02 \mathrm{~mol})$ and terephthalaldehyde $(0.01 \mathrm{~mol})$ were stirred in ethanol (10-20 $\mathrm{mL}$ ) for $2-3 \mathrm{~h}$ and $10 \mathrm{~mL} 40 \%$ sodium hydroxide solution was added drop wise with constant stirring at room temp till a light brown mass was obtained. Then the mixture was kept overnight at room temperature and the contents were poured on crushed ice and acidified with dilute hydrochloric acid, which resulted in the precipitation of chalcone. The crude $3,3^{\prime}$-(1,4-phenylene) bis(1-(2,4-dichlorophenyl)prop-2-en-1-one) was filtered, dried and recrystallized from methanol $[18,19]$.
Step ii: Synthesis of 6,6'-(1,4-phenylene)bis(4-(2,4-dichlorophenyl)pyrimidin-2-amine) (Int-II)

To a mixture of 3,3'-(1,4-phenylene)bis(1-(2,4-dichlorophenyl)prop-2-en-1-one) (0.01 mol) (synthesized in previous step- $i)$ and potassium hydroxide $(0.01 \mathrm{~mol})$ in $80 \mathrm{~mL}$ absolute ethanol, $40 \mathrm{~mL} 0.50 \mathrm{M}$ solution of guanidine hydrochloride in ethanol was added. After addition, the mixture was refluxed for $4-5 \mathrm{~h}\left(50{ }^{\circ} \mathrm{C}\right)$. The progress of reaction was monitored by TLC and the reaction mixture was cooled at room temperature and quenched with $20 \mathrm{~mL}$ of $0.5 \mathrm{M}$ solution of hydrochloric acid in water. The reaction mixture was shaken to ensure mixing and concentrated to obtain solid which was recrystallized from ethanol [18].

Step iii: Synthesis of final compounds (s1-s18)

A mixture of 6,6'-(1,4-phenylene)bis(4-(2,4-dichlorophenyl)pyrimidin-2-amine) (0.01 mol) (synthesized in previous step-ii) and substituted aldehyde $(0.02 \mathrm{~mol})$ were 
Table 3 Anticancer activity results of the synthesized bispyrimidine molecules

\begin{tabular}{lcll}
\hline \multicolumn{3}{l}{ Anticancer activity $\left(\mathrm{IC}_{\mathbf{5 0}}=\boldsymbol{\mu \mathrm { mol } / \mathrm { mL } )}\right.$} & \\
\hline Compound no. & $\begin{array}{l}\text { Cancer cell } \\
\text { line (HCT-116) }\end{array}$ & Compound no. & $\begin{array}{l}\text { Cancer cell } \\
\text { line (HCT- } \\
\mathbf{1 1 6})\end{array}$ \\
\hline s1. & 12.56 & $\mathbf{s 1 0 .}$ & 5.08 \\
s2. & 5.16 & $\mathbf{s 1 1 .}$ & 2.96 \\
s3. & 1.16 & $\mathbf{s 1 2 .}$ & 4.44 \\
s4. & 13.16 & $\mathbf{s 1 3 .}$ & 4.27 \\
s5. & 11.79 & $\mathbf{s 1 4 .}$ & 3.77 \\
s6. & 12.22 & $\mathbf{s 1 5 .}$ & 2.63 \\
s7. & 6.72 & $\mathbf{s 1 6 .}$ & 2.18 \\
s8. & 4.91 & $\mathbf{s 1 7 .}$ & 2.64 \\
s9. & 3.81 & $\mathbf{s 1 8 .}$ & 3.59 \\
5-Fluorouracil & 0.83 & $\mathbf{5 - F l u o r o u r a c i l}$ & 0.83
\end{tabular}

HCT-116 human colorectal carcinoma

refluxed in minimum amount of ethanol in presence of small amount of glacial acetic acid as catalyst for $2-3 \mathrm{~h}$ $\left(40{ }^{\circ} \mathrm{C}\right)$. The progress of reaction was monitored by TLC plates. The mixture was cooled and poured in ice cold water. The precipitated solid (different yellow color) was filtered and recrystallized with methanol.

\section{In vitro antimicrobial assay}

The in vitro antimicrobial study of the synthesized 6,6'-(1,4-phenylene)bis(4-(2,4-dichlorophenyl)pyrimidin2-amine) molecules was evaluated against Gram positive bacteria [Staphylococcus aureus (ATCC 11632) and Bacillus cereus (MTCC 7350)], Gram negative bacteria [Escherichia coli ATCC 35218, Pseudomonas aeruginosa
(ATCC 23564), Salmonella typhi (ATCC 15499) and Providencia rettgeri (MTCC-8099)] and fungal strainsAspergillus niger (MTCC-281); Aspergillus fumigatus (2483); Aspergillus flavus (1783) by tube dilution method [20]. The stock solution was prepared for the test compounds (s1-s18) and reference drugs (cefadroxil and fluconazole) in acetone to get a concentration of $100 \mu \mathrm{g} / \mathrm{mL}$ and this stock solution was used for further tube dilution with six concentration of 20,10,5.0, 2.5, 1.25, $0.625 \mu \mathrm{g} /$ $\mathrm{mL}$ for the antimicrobial study [21]. Dilution of test and standard compounds were prepared double strength nutrient broth-I.P (antibacterial) and sabouraud dextrose broth-I.P (antifungal). The samples were incubated at $37 \pm 1{ }^{\circ} \mathrm{C}$ for $24 \mathrm{~h}$ (bacteria), $25 \pm 1{ }^{\circ} \mathrm{C}$ for 7 days (A. niger), $30 \pm 1{ }^{\circ} \mathrm{C}$ for 15 days (A. flavus), $35 \pm 1{ }^{\circ} \mathrm{C}$ for $72 \mathrm{~h}$ (A. fumigates) respectively and results were recorded in terms of MIC (The lowest concentration of test substances which inhibited microbial growth) are presented in the Table 2.

\section{In vitro anticancer assay}

The in vitro cytotoxicity screening of synthesized 6,6'-(1,4-phenylene)bis(4-(2,4-dichlorophenyl)pyrimidin-2-amine) molecules was determined against human colorectal carcinoma [HCT-116 (ATCC (American Type Culture Collection) CCL-247)] cancer cell line using Sulforhodamine-B (SRB) assay. In this study, the cells were fixed with trichloroacetic acid and then stained with $0.4 \%(\mathrm{w} / \mathrm{v})$ Sulforhodamine B mixed with $1 \%$ acetic acid. Unbound dye was discarded by five washes of $1 \%$ acetic acid solution and protein-bound dye was solublised with $10 \mathrm{mM}$ Tris base for confirmation of optical density in a computer-interfaced, 96-well microtiter plate reader. The anticancer activity results recorded in $\mathrm{IC}_{50}$ value [22].

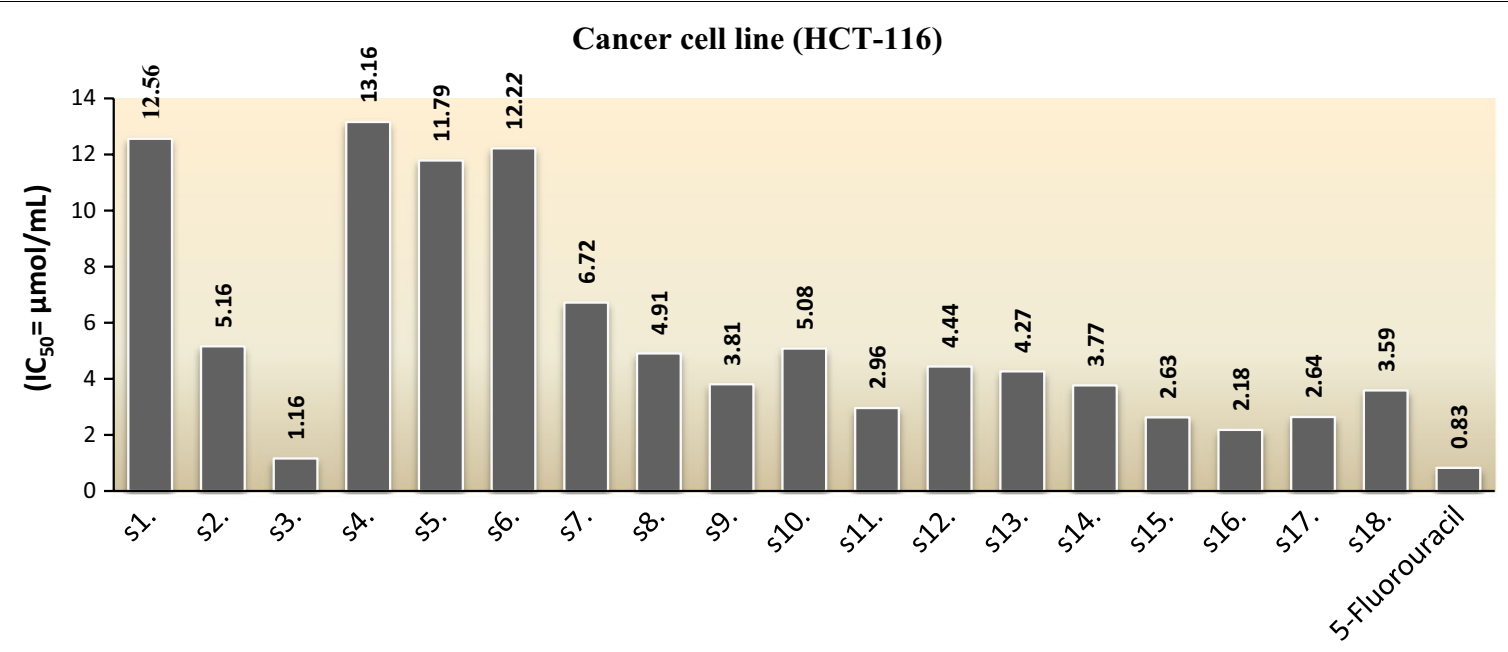

Fig. 3 Anticancer screening results of synthesized molecules against cancer cell line 


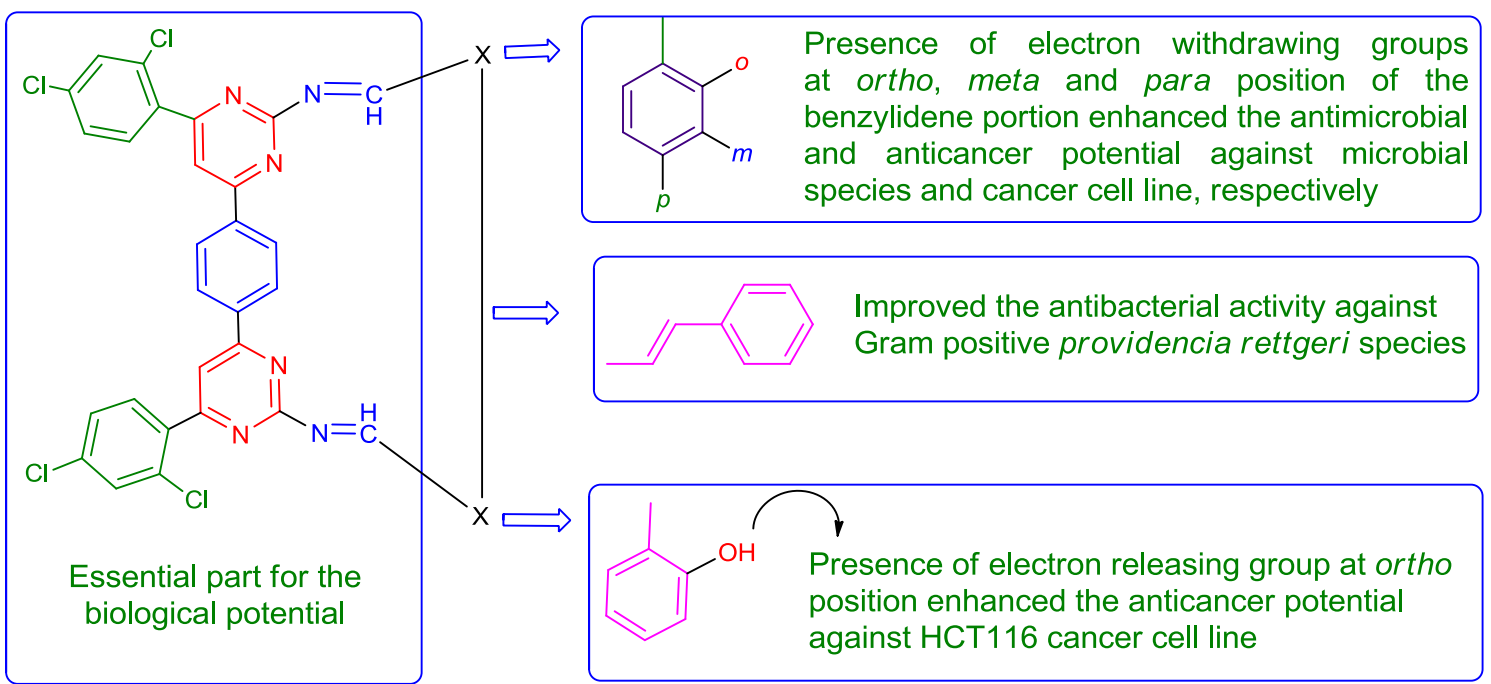

Fig. 4 Structural requirements for the antimicrobial and anticancer activities of synthesized bis-pyrimidine molecules

\section{Spectral characteristics of the synthesized pyrimidine compounds (s1-s18) \\ 6,6'-(1,4-Phenylene) \\ bis( $N$-(4-chlorobenzylidene)-4-(2,4-dichlorophenyl) \\ pyrimidin-2-amine) (s1)}

IR ( $\left.\mathrm{KBr}, \mathrm{cm}^{-1}\right)$ : 3088.93 (C-H str.), 1596.06 ( $\mathrm{C}=\mathrm{C}$ str.), $1665.82(\mathrm{~N}=\mathrm{CH}$ str.), $1332.11(\mathrm{C}-\mathrm{N}$ str.), $735.72(\mathrm{Ar}-\mathrm{Cl})$; MS ES + (ToF): $m / z 797\left[\mathrm{M}^{+}+1\right] ;{ }^{1} \mathrm{H}-\mathrm{NMR}(\delta$, DMSO$\left.d_{6}\right)$ : 7.36-7.86 (m, 18H, Ar-H), $10.05(\mathrm{~s}, 2 \mathrm{H}, \mathrm{N}=\mathrm{CH})$, $10.22\left(\mathrm{~s}, 2 \mathrm{H},(\mathrm{CH})_{2}\right.$ of pyrimidine ring); ${ }^{13} \mathrm{C}-\mathrm{NMR}(\delta$, DMSO- $\left.d_{6}\right): 160.2,145.7,138.9,136.2,131.2,130.2,129.98$, 129.94, 128.17, 128.13, 127.6; CHN: Calc. $\mathrm{C}_{40} \mathrm{H}_{22} \mathrm{Cl}_{6} \mathrm{~N}_{6}$ : C, 60.10; H, 2.77; N, 10.51; Found: C, 60.18; H, 2.71; N, 10.56 .

\section{4,4'-((6,6'-(1,4-Phenylene)bis(4-(2,4-dichloro-} phenyl)pyrimidine-6,2-diyl))bis(azanylylidene)) bis(methanylylidene))bis(2-ethoxyphenol) (s2) IR (KBr, cm $\left.{ }^{-1}\right)$ : 2978.51 (C-H str.), 1595.22 (C=C str.), $1665.80(\mathrm{~N}=\mathrm{CH}$ str.), 1331.51 (C-N str.), $73616(\mathrm{Ar}-\mathrm{Cl})$, $1104.41\left(\mathrm{C}-\mathrm{O}-\mathrm{C}_{2} \mathrm{H}_{5}\right.$, aralkyl ether), $3345.04(\mathrm{O}-\mathrm{H}$ str.); MS ES + (ToF): $m / z 849\left[\mathrm{M}^{+}+1\right]$; ${ }^{1} \mathrm{H}-\mathrm{NMR}(\delta$, DMSO$\left.d_{6}\right)$ : 6.78-8.27 (m, 16H, Ar-H), $9.70(\mathrm{~s}, 2 \mathrm{H}, \mathrm{N}=\mathrm{CH})$, $10.03\left(\mathrm{~s}, 2 \mathrm{H},(\mathrm{CH})_{2}\right.$ of pyrimidine ring), $3.98(\mathrm{t}, 4 \mathrm{H}$, $\left.\left(\mathrm{CH}_{2}\right)_{2}\right), 1.32\left(\mathrm{~d}, 6 \mathrm{H},\left(\mathrm{CH}_{3}\right)_{2}\right) ;{ }^{13} \mathrm{C}-\mathrm{NMR}\left(\delta\right.$, DMSO- $\left.d_{6}\right)$ : $165.67,164.08,153.58,147.66,136.66,137.17,132.63$, $132.57,130.48,129.48,129.91,128.84,126.44,112.44$, 107.36, 64.4, 14.8; CHN: Calc. $\mathrm{C}_{44} \mathrm{H}_{32} \mathrm{Cl}_{4} \mathrm{~N}_{6} \mathrm{O}_{4}$ : C, 62.13; H, 3.79; N, 9.88; Found: C, 62.10; H, 3.68; N, 9.81.

\section{6,6'-(1,4-Phenylene)bis(4-(2,4-dichlorophenyl)-N-(3-nitr obenzylidene)pyrimidin-2-amine) (s3)}

IR (KBr, cm $\left.{ }^{-1}\right)$ : 3088.09 (C-H str.), $1595.51 \quad(\mathrm{C}=\mathrm{C}$ str.), 1665.33 ( $\mathrm{N}=\mathrm{CH}$ str.), 1337.08 (C-N str.), 734.25
$(\mathrm{Ar}-\mathrm{Cl}) ; 1373.82\left(\mathrm{C}-\mathrm{NO}_{2}\right.$, str., $\left.\mathrm{NO}_{2}\right) ; \mathrm{MS} \mathrm{ES}+(\mathrm{ToF})$ : $m / z 819\left[\mathrm{M}^{+}+1\right] ;{ }^{1} \mathrm{H}-\mathrm{NMR}\left(\delta, \mathrm{DMSO}-d_{6}\right): 7.42-8.34(\mathrm{~m}$, $18 \mathrm{H}, \mathrm{Ar}-\mathrm{H}), 10.04(\mathrm{~s}, 2 \mathrm{H}, \mathrm{N}=\mathrm{CH}), 10.15\left(\mathrm{~s}, 2 \mathrm{H},(\mathrm{CH})_{2}\right.$ of pyrimidine ring); ${ }^{13} \mathrm{C}-\mathrm{NMR}\left(\delta\right.$, DMSO- $\left.d_{6}\right): 165.56$, $164.35,145.90,138.92,137.86,137.49,136.22,135.37$, $131.90,130.49,129.49,129.88,128.21,127.61,124.45$; CHN: Calc. $\mathrm{C}_{40} \mathrm{H}_{22} \mathrm{Cl}_{4} \mathrm{~N}_{8} \mathrm{O}_{4}$ : C, 58.56; H, 2.70; N, 13.66; Found: C, 58.50; H, 2.73; N, 13.70 .

\section{4,4'-((6,6'-(1,4-Phenylene)bis(4-(2,4-dichloro-} phenyl)pyrimidine-6,2-diyl))bis(azanylylidene)) bis(methanylylidene))diphenol (s4)

IR ( $\left.\mathrm{KBr}, \mathrm{cm}^{-1}\right)$ : 3028.00 (C-H str.), 1595.49 (C=C str.), $1664.04(\mathrm{~N}=\mathrm{CH}$ str.), $1332.80(\mathrm{C}-\mathrm{N}$ str.), $735.82(\mathrm{Ar}-\mathrm{Cl})$, 3461.39 (O-H str.); MS ES + (ToF): $m / z 761\left[\mathrm{M}^{+}+1\right]$; ${ }^{1} \mathrm{H}-\mathrm{NMR}\left(\delta\right.$, DMSO- $\left.d_{6}\right): 7.33-8.34(\mathrm{~m}, 18 \mathrm{H}, \mathrm{Ar}-\mathrm{H}), 9.79$ (s, $2 \mathrm{H}, \mathrm{N}=\mathrm{CH}), 10.10\left(\mathrm{~s}, 2 \mathrm{H},(\mathrm{CH})_{2}\right.$ of pyrimidine ring), 6.92 (s, $2 \mathrm{H}, \mathrm{Ar}-\mathrm{OH}) ;{ }^{13} \mathrm{C}-\mathrm{NMR}\left(\delta, \mathrm{DMSO}-d_{6}\right): 165.56$, $164.36,136.45,136.40,134.93,130.24,130.18,129.94$, 129.05, 128.03, 127.62, 116.30, 107.26; CHN: Calc. $\mathrm{C}_{40} \mathrm{H}_{24} \mathrm{Cl}_{4} \mathrm{~N}_{6} \mathrm{O}_{2}:$ C, 63.01; H, 3.17; N, 11.02; Found: C, $63.05 ; \mathrm{H}, 3.19 ; \mathrm{N}, 11.08$.

\section{6,6'-(1,4-Phenylene)bis(4-(2,4-dichlorophenyl)- $N$-(3,4-di} methoxybenzylidene)pyrimidin-2-amine) (s5)

IR ( $\left.\mathrm{KBr}, \mathrm{cm}^{-1}\right)$ : 3027.69 (C-H str.), 1596.45 (C=C str.), 1663.75 ( $\mathrm{N}=\mathrm{CH}$ str.), 1332.00 (C-N str.), 735.35 ( $\mathrm{Ar}-\mathrm{Cl}$ ), $3088.33\left(\mathrm{C}-\mathrm{O}-\mathrm{CH}_{3}\right.$, aralkyl ether); MS ES + (ToF): $m / z$ $849\left[\mathrm{M}^{+}+1\right] ;{ }^{1} \mathrm{H}-\mathrm{NMR}\left(\delta\right.$, DMSO- $\left.d_{6}\right)$ : 7.01-8.34 $(\mathrm{m}, 16 \mathrm{H}$, $\mathrm{Ar}-\mathrm{H}), 9.85(\mathrm{~s}, 2 \mathrm{H}, \mathrm{N}=\mathrm{CH}), 10.10\left(\mathrm{~s}, 2 \mathrm{H},(\mathrm{CH})_{2}\right.$ of pyrimidine ring), $3.84\left(\mathrm{~s}, 12 \mathrm{H},\left(\mathrm{OCH}_{3}\right)_{4}\right) ;{ }^{13} \mathrm{C}-\mathrm{NMR}(\delta$, DMSO$\left.d_{6}\right)$ : $165.65,164.36,137.87,136.95,134.93,134.93,131.83$, $130.43,129.95,128.22,128.17,127.62,111.82,107.25$, 
56.38; CHN: Calc. $\mathrm{C}_{44} \mathrm{H}_{32} \mathrm{Cl}_{4} \mathrm{~N}_{6} \mathrm{O}_{4}$ : C, 62.13; $\mathrm{H}, 3.79 ; \mathrm{N}$, 9.88; Found: C, 62.17; H, 3.75; N, 9.92.

\section{6,6'-(1,4-Phenylene)bis(4-(2,4-dichlorophenyl)- $N$-(2-nitr obenzylidene)pyrimidin-2-amine) (s6)}

IR ( $\left.\mathrm{KBr}, \mathrm{cm}^{-1}\right)$ : 3028.75 (C-H str.), 1595.16 ( $\mathrm{C}=\mathrm{C}$ str.), 1664.50 ( $\mathrm{N}=\mathrm{CH}$ str.), 1333.19 (C-N str.), 736.40 (Ar$\mathrm{Cl}) ; 1372.97\left(\mathrm{C}-\mathrm{NO}_{2}\right.$ sym. str., $\left.\mathrm{NO}_{2}\right)$; $\mathrm{MS} \mathrm{ES}+(\mathrm{ToF})$ : $m / z 819\left[\mathrm{M}^{+}+1\right] ;{ }^{1} \mathrm{H}-\mathrm{NMR}\left(\delta, \mathrm{DMSO}-d_{6}\right): 7.02-8.34(\mathrm{~m}$, 18H, Ar-H), 10.00 (s, 2H, N=CH), 10.10 (s, 2H, $(\mathrm{CH})_{2}$ of pyrimidine ring); ${ }^{13} \mathrm{C}-\mathrm{NMR}\left(\delta, \mathrm{DMSO}-d_{6}\right)$ : 165.55 , $164.36,142.36,137.87,134.93,132.66,131.37,130.27$, 130.22, 129.99, 129.98, 128.16, 128.13, 127.61, 107.26; CHN: Calc. $\mathrm{C}_{40} \mathrm{H}_{22} \mathrm{Cl}_{4} \mathrm{~N}_{8} \mathrm{O}_{4}$ : C, 58.56; H, 2.70; N, 13.66; Found: C, 58.60; H, 2.74; N, 13.69 .

\section{6,6'-(1,4-Phenylene)bis(4-(2,4-dichlorophenyl)-N-(4-nitr} obenzylidene)pyrimidin-2-amine) (s7)

IR ( $\mathrm{KBr}, \mathrm{cm}^{-1}$ ): 3088.00 (C-H str.), 1595.42 ( $\mathrm{C}=\mathrm{C}$ str.), 1664.00 ( $\mathrm{N}=\mathrm{CH}$ str.), 1334.07 (C-N str.), 735.84 (Ar$\mathrm{Cl}), 1372.55\left(\mathrm{C}-\mathrm{NO}_{2}\right.$ sym. str., $\left.\mathrm{NO}_{2}\right)$; $\mathrm{MS} \mathrm{ES}+(\mathrm{ToF})$ : $m / z 819\left[\mathrm{M}^{+}+1\right] ;{ }^{1} \mathrm{H}-\mathrm{NMR}\left(\delta, \mathrm{DMSO}-d_{6}\right): 7.02-8.34(\mathrm{~m}$, $18 \mathrm{H}, \mathrm{Ar}-\mathrm{H}), 10.00$ (s, 2H, N=CH), 10.10 (s, 2H, $(\mathrm{CH})_{2}$ of pyrimidine ring); ${ }^{13} \mathrm{C}-\mathrm{NMR}\left(\delta, \mathrm{DMSO}-d_{6}\right)$ : 165.55 , $164.37,163.17,145.88,142.74,140.07,137.86,136.94$, 134.93, 132.79, 131.84, 130.27, 129.94, 128.16, 128.10, 124.71, 107.1; CHN: Calc. $\mathrm{C}_{40} \mathrm{H}_{22} \mathrm{Cl}_{4} \mathrm{~N}_{8} \mathrm{O}_{4}: \mathrm{C}, 58.56 ; \mathrm{H}$, 2.70; N, 13.66; Found: C, 58.61; H, 2.76; N, 13.70 .

6,6'-(1,4-Phenylene)bis(4-(2,4-dichlorophenyl)-N-(4-(dimet hylamino)benzylidene)pyrimidin-2-amine) (s8)

IR ( $\mathrm{KBr}, \mathrm{cm}^{-1}$ ): 3028.00 (C-H str.), 1590.10 ( $\mathrm{C}=\mathrm{C}$ str.), $1662.86(\mathrm{~N}=\mathrm{CH}$ str.), 1331.98 (C-N str.), 733.88 (Ar$\mathrm{Cl}), 2826.15$ (C-H str., $\left.-\mathrm{CH}_{3}\right)$; MS ES + (ToF): $m / z 815$ $\left[\mathrm{M}^{+}+1\right]$; ${ }^{1} \mathrm{H}-\mathrm{NMR}\left(\delta, \mathrm{DMSO}-d_{6}\right): 6.77-8.34(\mathrm{~m}, 18 \mathrm{H}$, Ar-H), $9.67(\mathrm{~s}, 2 \mathrm{H}, \mathrm{N}=\mathrm{CH}), 10.04\left(\mathrm{~s}, 2 \mathrm{H},(\mathrm{CH})_{2}\right.$ of pyrimidine ring), $3.04\left(\mathrm{~s}, 12 \mathrm{H},\left(\mathrm{CH}_{3}\right)_{4}\right) ;{ }^{13} \mathrm{C}-\mathrm{NMR}(\delta$, DMSO$\left.d_{6}\right)$ : 165.54, 164.36, 145.70, 142.74, 137.87, 136.40, 134.94, 131.37, 130.27, 130.18, 129.93, 128.16, 127.60, 111.54, 107.26, 40.15; CHN: Calc. $\mathrm{C}_{44} \mathrm{H}_{34} \mathrm{Cl}_{4} \mathrm{~N}_{8}$ : C, 64.72; H, 4.20; N, 13.72; Found: C, 64.76; H, 4.26; N, 13.74 .

6,6'-(1,4-Phenylene)bis(4-(2,4-dichlorophenyl)- $N$-(3-methoxybenzylidene)pyrimidin-2-amine) (s9)

IR ( $\mathrm{KBr}, \mathrm{cm}^{-1}$ ): 3028.79 (C-H str.), 1595.05 ( $\mathrm{C}=\mathrm{C}$ str.), 1664.77 ( $\mathrm{N}=\mathrm{CH}$ str.), 1332.13 (C-N str.), 736.46 (Ar$\mathrm{Cl}), 3089.60\left(\mathrm{C}-\mathrm{O}-\mathrm{CH}_{3}\right.$, aralkyl ether); MS ES + (ToF): $m / z 789\left[\mathrm{M}^{+}+1\right] ;{ }^{1} \mathrm{H}-\mathrm{NMR}\left(\delta, \mathrm{DMSO}-d_{6}\right): 7.03-8.34(\mathrm{~m}$, $18 \mathrm{H}, \mathrm{Ar}-\mathrm{H}), 10.04$ (s, 2H, N=CH), 10.10 (s, 2H, $(\mathrm{CH})_{2}$ of pyrimidine ring), $3.84\left(\mathrm{~s}, 6 \mathrm{H},\left(\mathrm{OCH}_{3}\right)_{2}\right) ;{ }^{13} \mathrm{C}-\mathrm{NMR}$ $\left(\delta\right.$, DMSO- $\left.d_{6}\right): 165.55,164.36,145.86,137.86,136.94$, $134.93,139.79,130.94,129.99,128.19,128.12,127.61$,
116.6, 109.26, 56.8; CHN: Calc. $\mathrm{C}_{42} \mathrm{H}_{28} \mathrm{Cl}_{4} \mathrm{~N}_{6} \mathrm{O}_{2}$ : C, 63.81; H, 3.57; N, 10.63; Found: C, 63.85; H, 3.60; N, 10.68 .

6,6'-(1,4-Phenylene)bis(4-(2,4-dichlorophenyl)-N-(4-methoxybenzylidene)pyrimidin-2-amine) (s10)

IR ( $\mathrm{KBr}, \mathrm{cm}^{-1}$ ): 3027.89 (C-H str.), 1595.38 (C=C str.), 1664.37 ( $\mathrm{N}=\mathrm{CH}$ str.), 1332.40 (C-N str.), 736.36 (Ar$\mathrm{Cl}), 3088.96\left(\mathrm{C}-\mathrm{O}-\mathrm{CH}_{3}\right.$, aralkyl ether); MS ES + (ToF): $m / z 789\left[\mathrm{M}^{+}+1\right] ;{ }^{1} \mathrm{H}-\mathrm{NMR}\left(\delta, \mathrm{DMSO}-d_{6}\right): 7.02-8.34(\mathrm{~m}$, $18 \mathrm{H}, \mathrm{Ar}-\mathrm{H}), 10.05$ (s, 2H, N=CH), $10.10\left(\mathrm{~s}, 2 \mathrm{H},(\mathrm{CH})_{2}\right.$ of pyrimidine ring), $3.84\left(\mathrm{~s}, 6 \mathrm{H},\left(\mathrm{OCH}_{3}\right)_{2}\right) ;{ }^{13} \mathrm{C}-\mathrm{NMR}$ $\left(\delta\right.$, DMSO- $\left.d_{6}\right)$ : 165.57, 164.37, 163.50, 145.88, 139.94, $134.93,132.73,130.4,129.88,128.16,128.13,127.96$, 114.9, 108.0, 52.2; CHN: Calc. $\mathrm{C}_{42} \mathrm{H}_{28} \mathrm{Cl}_{4} \mathrm{~N}_{6} \mathrm{O}_{2}$ : C, 63.81; H, 3.57; N, 10.63; Found: C, 63.85; H, 3.61; N, 10.70 .

6,6'-(1,4-Phenylene)bis( $N$-(2,4-dichlorobenzylidene)-4-(2,4 -dichlorophenyl)pyrimidin-2-amine) (s11)

IR ( $\left.\mathrm{KBr}, \mathrm{cm}^{-1}\right)$ : 3025.92 (C-H str.), 1594.64 ( $\mathrm{C}=\mathrm{C}$ str.), 1663.17 ( $\mathrm{N}=\mathrm{CH}$ str.), 1330.99 (C-N str.), 734.93 (Ar-Cl); MS ES + (ToF): $m / z 865\left[\mathrm{M}^{+}+1\right] ;{ }^{1} \mathrm{H}-\mathrm{NMR}(\delta$, DMSO$\left.d_{6}\right)$ : 7.28-8.02 (m, 16H, Ar-H), $10.02(\mathrm{~s}, 2 \mathrm{H}, \mathrm{N}=\mathrm{CH})$, $10.10\left(\mathrm{~s}, 2 \mathrm{H},(\mathrm{CH})_{2}\right.$ of pyrimidine ring); ${ }^{13} \mathrm{C}-\mathrm{NMR}(\delta$, DMSO- $\left.d_{6}\right)$ : $165.59,164.38,163.52,145.87,137.80$, $136.92,137.78,131.78,130.94,130.27,129.80,128.02$, 128.05, 127.50, 100.00; CHN: Calc. $\mathrm{C}_{40} \mathrm{H}_{20} \mathrm{Cl}_{8} \mathrm{~N}_{6}: \mathrm{C}$, 55.33; H, 2.32; N, 9.68; Found: C, 55.338; H, 2.37; N, 9.72.

6,6'-(1,4-Phenylene)bis(4-(2,4-dichlorophenyl)-N-(2-methoxybenzylidene)pyrimidin-2-amine) (s12)

IR ( $\left.\mathrm{KBr}, \mathrm{cm}^{-1}\right)$ : 3027.43 (C-H str.), 1592.13 (C=C str.), 1663.49 ( $\mathrm{N}=\mathrm{CH}$ str.), 1332.08 (C-N str.), 3088.50 (C-O$\mathrm{CH}_{3}$, aralkyl ether), 735.94 (Ar-Cl); MS ES + (ToF): $m / z$ $789\left[\mathrm{M}^{+}+1\right] ;{ }^{1} \mathrm{H}-\mathrm{NMR}\left(\delta, \mathrm{DMSO}-d_{6}\right): 6.96-7.87(\mathrm{~m}, 18 \mathrm{H}$, Ar-H), $9.20(\mathrm{~s}, 2 \mathrm{H}, \mathrm{N}=\mathrm{CH}), 10.02\left(\mathrm{~s}, 2 \mathrm{H},(\mathrm{CH})_{2}\right.$ of pyrimidine ring), $3.85\left\{\mathrm{~s}, 6 \mathrm{H},\left(\mathrm{OCH}_{3}\right)_{2}\right\} ;{ }^{13} \mathrm{C}-\mathrm{NMR}(\delta$, DMSO$\left.d_{6}\right): 165.59,164.37,163.50,145.88,139.90,135.98,134.93$, 132.73, 130.4, 129.80, 128.19, 128.13, 127.96, 115.9, 108.0, 55.2; CHN: Calc. $\mathrm{C}_{42} \mathrm{H}_{28} \mathrm{Cl}_{4} \mathrm{~N}_{6} \mathrm{O}_{2}$ : C, 63.81; H, 3.57; N, 10.63; Found: C, 63.85; H, 3.61; N, 10.68.

\section{4,4'-((6,6'-(1,4-Phenylene)bis(4-(2,4-dichloro-}

phenyl)pyrimidine-6,2-diyl))bis(azanylylidene)) bis(methanylylidene))bis(2-methoxyphenol) (s13)

IR (KBr, $\left.\mathrm{cm}^{-1}\right)$ : 3027.22 (C-H str.), $1596.30 \quad(\mathrm{C}=\mathrm{C}$ str.), 1663.95 ( $\mathrm{N}=\mathrm{CH}$ str.), 1331.37 ( $\mathrm{C}-\mathrm{N}$ str.), 3461.41 (O-H str.), 3088.44 (C-O- $\mathrm{CH}_{3}$, aralkyl ether), 736.07 $(\mathrm{Ar}-\mathrm{Cl}) ; \mathrm{MS} \mathrm{ES}+(\mathrm{ToF}): m / z 821\left[\mathrm{M}^{+}+1\right] ;{ }^{1} \mathrm{H}-\mathrm{NMR}$ $\left(\delta\right.$, DMSO- $\left.d_{6}\right): 7.03-8.34(\mathrm{~m}, 16 \mathrm{H}, \mathrm{Ar}-\mathrm{H}), 10.04(\mathrm{~s}, 2 \mathrm{H}$, $\mathrm{N}=\mathrm{CH}), 10.10\left(\mathrm{~s}, 2 \mathrm{H},(\mathrm{CH})_{2}\right.$ of pyrimidine ring), $3.85\{\mathrm{~s}$, $\left.6 \mathrm{H},\left(\mathrm{OCH}_{3}\right)_{2}\right\} ;{ }^{13} \mathrm{C}-\mathrm{NMR}\left(\delta\right.$, DMSO- $\left.d_{6}\right): 165.55,164.36$, $151.01,149.01,137.71,136.94,134.93,132.78,131.84$, $130.94,130.34,129.88,128.16,128.05,107.61,61.35$; 
CHN: Calc. $\mathrm{C}_{42} \mathrm{H}_{28} \mathrm{Cl}_{4} \mathrm{~N}_{6} \mathrm{O}_{4}$ : C, 61.33; H, 3.43; N, 10.22; Found: C, 61.38; H, 3.48; N, 10.27 .

\section{6,6'-(1,4-Phenylene)bis( $N$-(2-chlorobenzylidene)-4-(2,4-di chlorophenyl)pyrimidin-2-amine) (s14)}

IR ( $\left.\mathrm{KBr}, \mathrm{cm}^{-1}\right)$ : 2973.44 (C-H str.), 1599.67 (C=C str.), 1666.78 ( $\mathrm{N}=\mathrm{CH}$ str.), 1329.19 (C-N str.), 750.40 ( $\mathrm{Ar}-\mathrm{Cl})$; MS ES + (ToF): $m / z 797\left[\mathrm{M}^{+}+1\right] ;{ }^{1} \mathrm{H}-\mathrm{NMR}(\delta$, DMSO$\left.d_{6}\right)$ : 7.24-8.00 (m, 18H, Ar-H), $9.01(\mathrm{~s}, 2 \mathrm{H}, \mathrm{N}=\mathrm{CH})$, $10.10\left(\mathrm{~s}, 2 \mathrm{H},(\mathrm{CH})_{2}\right.$ of pyrimidine ring); ${ }^{13} \mathrm{C}-\mathrm{NMR}(\delta$, DMSO- $\left.d_{6}\right): 165.65,164.35,162.50,146.75,136.24,134.37$, $131.85,130.31,130.22,130.18,129.90,129.20,128.03$, 120.02, 128.00, 127.88, 100.39; CHN: Calc. $\mathrm{C}_{40} \mathrm{H}_{22} \mathrm{Cl}_{6} \mathrm{~N}_{6}$ : C, 60.10; H, 2.77; N, 10.51; Found: C, 60.17; H, 2.80; N, 10.55 .

\section{2,2'-(((6,6'-(1,4-Phenylene)bis(4-(2,4-dichloro-} phenyl)pyrimidine-6,2-diyl))bis(azanylylidene)) bis(methanylylidene))diphenol (s15)

IR ( $\left.\mathrm{KBr}, \mathrm{cm}^{-1}\right)$ : 2972.97 (C-H str.), 1598.70 ( $\mathrm{C}=\mathrm{C}$ str.), 1698.99 ( $\mathrm{N}=\mathrm{CH}$ str.), 1330.19 (C-N str.), 750.53 ( $\mathrm{Ar}-\mathrm{Cl}$ ); 3360.91 (O-H str.); MS ES + (ToF): $m / z 761\left[\mathrm{M}^{+}+1\right]$; ${ }^{1} \mathrm{H}-\mathrm{NMR}\left(\delta, \mathrm{DMSO}-d_{6}\right): 7.27-7.99(\mathrm{~m}, 18 \mathrm{H}, \mathrm{Ar}-\mathrm{H}), 9.99$ (s, $2 \mathrm{H}, \mathrm{N}=\mathrm{CH}), 10.07\left(\mathrm{~s}, 2 \mathrm{H},(\mathrm{CH})_{2}\right.$ of pyrimidine ring); ${ }^{13} \mathrm{C}-\mathrm{NMR}\left(\delta\right.$, DMSO- $\left.d_{6}\right)$ : 165.55, 164.36, 137.78, 136.46, 130.31, 129.90, 128.93, 128.12, 117.08, 110.04; CHN: Calc. $\mathrm{C}_{40} \mathrm{H}_{24} \mathrm{Cl}_{4} \mathrm{~N}_{6} \mathrm{O}_{2}:$ C, 63.01; H, 3.17; N, 11.02; Found: C, 63.05; H, 3.19; N, 11.07 .

6,6'-(1,4-Phenylene)bis(4-(2,4-dichlorophenyl)- $N$-(4-(diethy lamino)benzylidene)pyrimidin-2-amine) (s16)

IR ( $\left.\mathrm{KBr}, \mathrm{cm}^{-1}\right)$ : 2974.01 (C-H str.), 1590.98 ( $\mathrm{C}=\mathrm{C}$ str.), 1695.19 ( $\mathrm{N}=\mathrm{CH}$ str.), 1352.37 (C-N str.), 750.16 ( $\mathrm{Ar}-$ Cl), $2826.51\left(\mathrm{C}-\mathrm{H}\right.$ str., $\left.-\mathrm{C}_{2} \mathrm{H}_{5}\right)$; MS ES + (ToF): $\mathrm{m} / z$ $871\left[\mathrm{M}^{+}+1\right]$; ${ }^{1} \mathrm{H}-\mathrm{NMR}\left(\delta\right.$, DMSO- $\left.d_{6}\right): 7.26-8.00(\mathrm{~m}$, $18 \mathrm{H}, \mathrm{Ar}-\mathrm{H}), 9.63(\mathrm{~s}, 2 \mathrm{H}, \mathrm{N}=\mathrm{CH}), 10.00\left(\mathrm{~s}, 2 \mathrm{H},(\mathrm{CH})_{2}\right.$ of pyrimidine ring), 3.38-3.49 \{q, $\left.8 \mathrm{H},\left(\mathrm{CH}_{2}\right)_{4}\right\}, 1.07-1.15\{\mathrm{t}$, $\left.12 \mathrm{H},\left(\mathrm{CH}_{3}\right)_{4}\right\} ;{ }^{13} \mathrm{C}-\mathrm{NMR}\left(\delta\right.$, DMSO- $\left.d_{6}\right): 167.55,164.36$, $159.36,136.42,131.86,130.30,130.18,129.91,128.94$, 127.49, 126.62, 124.49, 111.0, 44.43, 12.74; CHN: Calc. $\mathrm{C}_{48} \mathrm{H}_{42} \mathrm{Cl}_{4} \mathrm{~N}_{8}$ : C, 66.06; H, 4.85; N, 12.84; Found: C, 66.10; $\mathrm{H}, 4.90 ; \mathrm{N}, 12.88$.

\section{6,6'-(1,4-Phenylene)bis( $N$-(3-chlorobenzylidene)-4-(2,4-di} chlorophenyl)pyrimidin-2-amine) (s17)

IR ( $\left.\mathrm{KBr}, \mathrm{cm}^{-1}\right)$ : 2974.44 (C-H str.), 1579.02 ( $\mathrm{C}=\mathrm{C}$ str.), $1693.29(\mathrm{~N}=\mathrm{CH}$ str.), 1328.36 (C-N str.), 750.11 (Ar-Cl); MS ES + (ToF): $m / z 797\left[\mathrm{M}^{+}+1\right] ;{ }^{1} \mathrm{H}-\mathrm{NMR}(\delta$, DMSO$\left.d_{6}\right): 7.25-8.03(\mathrm{~m}, 18 \mathrm{H}, \mathrm{Ar}-\mathrm{H}), 10.00(\mathrm{~s}, 2 \mathrm{H}, \mathrm{N}=\mathrm{CH})$, $10.04\left(\mathrm{~s}, 2 \mathrm{H},(\mathrm{CH})_{2}\right.$ of pyrimidine ring); ${ }^{13} \mathrm{C}-\mathrm{NMR}(\delta$, DMSO- $\left.d_{6}\right): 165.55,164.36,136.91,131.85,131.77$,
$130.31,130.23,129.92,129.21,128.98,128.10,127.49$, 126.65, 100.90; CHN: Calc. $\mathrm{C}_{40} \mathrm{H}_{22} \mathrm{Cl}_{6} \mathrm{~N}_{6}$ : C, 60.10; H, 2.77; N, 10.51; Found: C, 60.15; H, 2.80; N, 10.48 .

\section{4-(2,4-Dichlorophenyl)-6-(4-(6-(2,4-dichlorophenyl)-2- (((E)-3-phenylallylidene)amino) pyrimidin-4-yl)} phenyl)- $N$-((E)-3-phenylallylidene)pyrimidin-2-amine (s18) IR ( $\left.\mathrm{KBr}, \mathrm{cm}^{-1}\right)$ : 2973.44 (C-H str.), 1597.13 (C=C str.), 1669.71 ( $\mathrm{N}=\mathrm{CH}$ str.), 1329.59 (C-N str.), 749.84 ( $\mathrm{Ar}-$ $\mathrm{Cl}), 2829.12$ (C-H str. aliphatic); ${ }^{1} \mathrm{H}-\mathrm{NMR}(\delta$, DMSO$\left.d_{6}\right)$ : 7.45-8.04 (m, 20H, Ar-H), 7.55 dd, 2H, $(\mathrm{CH})_{2}$ of $\mathrm{N}=\mathrm{CH}\}, 9.00\left(\mathrm{~s}, 2 \mathrm{H},(\mathrm{CH})_{2}\right.$ of pyrimidine ring), $6.86\{\mathrm{t}$, $\left.2 \mathrm{H},(\mathrm{CH})_{2}\right\}, 7.34\left\{\mathrm{~d}, 2 \mathrm{H},(\mathrm{CH})_{2}\right\} ;{ }^{13} \mathrm{C}-\mathrm{NMR}(\delta$, DMSO$\left.d_{6}\right): 167.89,164.23,163.9,135.6,134.69,133.56,130.20$, 130.87, 129.88, 128.00, 128.34, 127.02, 127.90, 120.12, 110.1; CHN: Calc. $\mathrm{C}_{44} \mathrm{H}_{28} \mathrm{Cl}_{4} \mathrm{~N}_{6}: \mathrm{C}, 67.53 ; \mathrm{H}, 3.61 ; \mathrm{N}$, 10.74; Found: C, 67.51; H, 3.68; N, 10.77.

\section{Conclusion}

In conclusion, we have described a simple and efficient protocol for the synthesis of new bis-pyrimidine molecules (s1-s18) with appreciable yields. The in vitro antibacterial, antifungal and anticancer potential of all the synthesized compounds were investigated. It is evident that synthesized compounds, s7, s8, s11, s14, s16, s17 and s18 have excellent antimicrobial activity and compound $\mathbf{s} 3$ exhibited good anticancer activity. For the above compounds a significant improvement in their antibacterial and antifungal activities has been examined over the earlier reported compounds. The 4,4'-(1,4-phenylene)bis(pyrimidin-2-amine) molecules reported have a probability to emerge as a valuable lead series with great potential to be used as antibacterial, antifungal and anticancer agents and as promising candidates for further efficacy evaluation.

\section{Authors' contributions}

$\mathrm{BN}$ and SK have designed, synthesized and carried out the antimicrobial activity and SML, KR, MV and SAAS have carried out the spectral analysis, interpretation and cytotoxicity study of synthesized compounds. All authors read and approved the final manuscript.

\footnotetext{
Author details

${ }^{1}$ Faculty of Pharmaceutical Sciences, Maharshi Dayanand University, Rohtak 124001, India. ${ }^{2}$ Faculty of Pharmacy, Universiti Teknologi MARA (UiTM), 42300 Bandar Puncak Alam, Selangor Darul Ehsan, Malaysia. ${ }^{3}$ Collaborative Drug Discovery Research (CDDR) Group, Pharmaceutical Life Sciences Community of Research, Universiti Teknologi MARA (UiTM), 40450 Shah Alam, Selangor Darul Ehsan, Malaysia. ${ }^{4}$ Department of Pharmacology and Toxicology, College of Pharmacy, Qassim University, Buraidah 51452, Saudi Arabia. ${ }^{5}$ Atta-ur-Rahman Institute for Natural Products Discovery (AuRIns), Universiti Teknologi MARA, Puncak Alam Campus, 42300 Bandar Puncak Alam, Selangor Darul Ehsan, Malaysia. ${ }^{6}$ Faculty of Pharmacy, Universiti Teknologi MARA (UiTM), Puncak Alam Campus, 42300 Bandar Puncak Alam, Selangor Darul Ehsan, Malaysia.
} 


\section{Acknowledgements}

The authors are thankful to Head, Department of Pharmaceutical Sciences, Maharshi Dayanand University, Rohtak, for providing necessary facilities to carry out this research work.

\section{Competing interests}

The authors declare that they have no competing interests.

\section{Availability of data and materials}

Provided in manuscript.

Ethics approval and consent to participate

Not applicable.

\section{Funding}

Not applicable.

\section{Publisher's Note}

Springer Nature remains neutral with regard to jurisdictional claims in published maps and institutional affiliations.

Received: 26 September 2017 Accepted: 13 February 2018

Published online: 25 June 2018

\section{References}

1. Anupama B, Dinda SC, Prasad YR, Rao AV (2012) Synthesis and antimicrobial activity of some new 2,4,6-trisubstituted pyrimidines. Int J Res Pharm Chem 2(2):231-236

2. Flefel EM, El-Sayed WA, Mohamed AM, El-Sofany WI, Awad HM (2017) Synthesis and anticancer activity of new 1-thia-4-azaspiro[4.5]decane, their derived thiazolopyrimidine and 1,3,4-thiadiazole thioglycosides. Molecules 22:1-13

3. Amer S, El-Wakiel N, El-Ghamry H (2013) Synthesis, spectral, antitumor and antimicrobial studies on Cu (II) complexes of purine and triazole Schiff base derivatives. J Mol Struct 1049:326-335

4. Xu X, Wang J, Yao Q (2015) Synthesis and quantitative structure-activity relationship (QSAR) analysis of some novel oxadiazolo[3,4-d]pyrimidine nucleosides derivatives as antiviral agents. Bioorg Med Chem Lett 25:241-244

5. Tozkoparan B, Ertan M, Kelicen P, Demirdamar R (1999) Synthesis and antiinflammatory activities of some thiazolo[3,2-a]pyrimidine derivatives. II Farmaco 54:588-593

6. Kumar D, Khan SI, Tekwani BL, Ponnan P, Rawat DS (2015) 4-Aminoquinoline-pyrimidine hybrids: synthesis, antimalarial activity, heme binding and docking studies. Eur J Med Chem 89:490-502

7. Chen Q, Zhu XL, Jiang LL, Liu ZM, Yang GF (2008) Synthesis, antifungal activity and CoMFA analysis of novel 1,2,4-triazolo[1,5-a]pyrimidine derivatives. Eur J Med Chem 43:595-603
8. El-Gaby MSA, Gaber AM, Atalla AA, AI-Wahab KAA (2002) Novel synthesis and antifungal activity of pyrrole and pyrrolo[2,3-d]pyrimidine derivatives containing sulfonamide moieties. II Farmaco 57:613-617

9. Hilmy KMH, Khalifa MMA, Hawata MAA, Keshk RMA, El-Torgman AA (2014) Synthesis of new pyrrolo[2,3-d]pyrimidine derivatives as antibacterial and antifungal agents. Eur J Med Chem 45:5243-5250

10. Liu Z, Wang Y, Lin H, Zuo D, Wang L, Zhao Y, Gong P (2014) Design, synthesis and biological evaluation of novel thieno[3,2-d]pyrimidine derivatives containing diaryl urea moiety as potent antitumor agents. Eur J Med Chem 85:215-227

11. Kurumurthy C, Rao PS, Swamy BV, Kumar GS, Rao PS, Narsaiah B, Velatooru LR, Pamanji R, Rao JV (2011) Synthesis of novel alkyltriazole tagged pyrido[2,3-d] pyrimidine derivatives and their anticancer activity. Eur J Med Chem 46:3462-3468

12. Xie F, Zhao H, Zhao L, Lou L, Hu Y (2009) Synthesis and biological evaluation of novel 2,4,5-substituted pyrimidine derivatives for anticancer activity. Bioorg Med Chem Lett 19:275-278

13. Jain SK, Chitre TS, Miniyar PB, Kathiravan MK, Bendre VS, Veer VS, Shahane SR, Shishoo CJ (2006) Biological and medicinal significance of pyrimidines. Curr Sci 90(6):793-803

14. Kaur N, Aggarwal AK, Sharma N, Choudhary B (2012) Synthesis and in vitro antimicrobial activity of pyrimidine derivatives. Int J Pharm Sci Drug Res 4(3):199-204

15. Fares M, Abou-Seri SM, Abdel-Aziz HA, Abbas SES, Youssef MM, Eladwy RA (2014) Synthesis and antitumor activity of pyrido[2,3-d]pyrimidine and pyrido[2,3-d] $[1,2,4]$ triazolo[4,3-a]pyrimidine derivatives that induce apoptosis through G1 cell-cycle arrest. Eur J Med Chem 83:155-166

16. Meenakshi K, Gopal N, Sarangapani M (2014) Synthesis, characterization and antimicrobial activity of some novel Schiff and Mannich bases of isatin. Int J Pharm Pharm Sci 6(6):318-322

17. Maddila S, Gorle S, Seshadri N, Lavanya P, Jonnalagadda SB (2016) Synthesis, antibacterial and antifungal activity of novel benzothiazole pyrimidine derivatives. Arab J Chem 9:681-687

18. Kumar S, Lim SM, Ramasamy K, Vasudevan M, Shah SAA, Selvaraj M, Narasimhan B (2017) Synthesis, molecular docking and biological evaluation of bis-pyrimidine Schiff base derivatives. Chem Cent J 11(89):1-16

19. Asiri AM, Khan SA (2011) Synthesis and anti-bacterial activities of a bis-chalcone, derived from thiophene and its bis-cyclized products. Molecules 16:523-531

20. Cappuccino JC, Sherman N (1999) Microbiology-a laboratory manual. Addison Wesley, California, p 263

21. Pharmacopoeia of India, vol I (2007) Controller of publication, ministry of health department, Govt. of India, New Delhi, pp 37

22. Skehan P, Storeng R, Scudiero D, Monks A, McMahon J, Vistica D, Warren JT, Bokesch H, Kenney S, Boyd MR (1990) New colorimetric cytotoxicity assay for anticancer-drug screening. J Natl Cancer Inst 82:1107-1112

\section{Submit your manuscript to a SpringerOpen ${ }^{\circ}$ journal and benefit from:}

- Convenient online submission

- Rigorous peer review

- Open access: articles freely available online

- High visibility within the field

- Retaining the copyright to your article

Submit your next manuscript at springeropen.com 